\title{
Can quantum correlations be completely quantum?
}

\author{
Eukasz Pankowski ${ }^{(1)}$ and Barbara Synak-Radtke ${ }^{(2)}$ \\ (1) Department of Mathematics Physics and Computer Science, University of Gdańsk, Poland and \\ ${ }^{(2)}$ Institute of Theoretical Physics and Astrophysics, University of Gdańsk, Poland
}

\begin{abstract}
Deficit of information zero-way was proposed in [1] as one of possible measures of quantumness of correlations. Numerical calculations suggested that there exist such states for which this quantity is almost equal to mutual information. In this paper we present a family of states for which we have equality between above measure of quantumness of correlations and the measure of total correlations - mutual information. It means that whole correlations in these states have, in some sense, quantum character and that quantum correlations do not necessarily imply classical correlations. We prove this intriguing feature for a subclass of $2 \otimes 2$ separable states. We also present numerical result suggesting that this interesting situation might also happen for $2 \otimes 2$ entangled states.
\end{abstract}

\section{INTRODUCTION}

It has been found in different contexts [1, 2, 3, 4, 5] that entanglement does not exhaust quantumness of correlations contained in compound quantum systems. In order to characterize quantumness of states the properly classically correlated bipartite states (shortly classically correlated) were defined [5]. These states can be written in the form

$$
\rho_{A B}=\sum_{i j} p_{i j}|i\rangle\langle i|\otimes| j\rangle\langle j|
$$

with coefficients $0 \leq p_{i j} \leq 1, \sum_{i j} p_{i j}=1 ;\{|i\rangle\}$ and $\{|j\rangle\}$ are local bases. The set of classically correlated states is invariant under local unitary operations. These states are diagonal in the so called biproduct basis. The non-classically correlated states are those that cannot be represented in the above form.

In 5] a measure of quantumness of correlations so called quantum deficit $\Delta$ was introduced, which is zero for classically correlated states (see extensive development [1]). In particular, it has been shown that there exist separable states which have nonzero deficit. This means that those separable states exhibit classical correlations between quantum properties. The paper [1] introduces variants of quantum deficit with restricted communication as independent candidates for the measure of quantumness of correlations. One of them called quantum deficit zero-way $\Delta^{\emptyset}$ is equivalent to the distance from classically correlated states [1]. Recently similar measures of quantumness of correlations based on the distance from classically correlated states has been introduced [6, 7] and quantumness of some subclasses of separable and entangled states was investigated.

The main purpose of the present paper is to inquire a relation between the measure of total correlations mutual information and quantum deficit. The later is defined [5] as the difference between informational content of a state and information that can be localized to a subsystem by use of local unitary operations and a dephasing channel. Quantum deficit refers us to this part of correlations that must be destroyed during the process of localizing information into a subsystem. In this paper we consider a special kind of quantum deficit called zero-way $\left(\Delta^{\emptyset}\right)[1]$ where only a restricted class, called $\mathcal{P}^{\emptyset}$, of protocols of localizing information is permitted. In $\mathcal{P}^{\emptyset}$ protocols we make local measurements and only after this we use a classical channel to collect data and then exploit classical correlations created by the measurements to localize information. Surprisingly, we find such states for which quantum deficit zero-way is equal to mutual information $I_{M}$ (the measure of total correlations in a state), i.e. $\Delta^{\emptyset}=I_{M}$. It means that all correlations in these states have quantum character. Notice that for maximally entangled states $\Delta^{\emptyset}=\frac{1}{2} I_{M}$, so we can say that only half of correlations manifest quantumness. In this context the $\Delta^{\emptyset}=I_{M}$ feature of the states presented in this paper seems especially interesting.

The notion of quantum deficit is built by use of quantities which has operational meaning in the regime of many copies: information $I$ is the number of pure states we can distill from a given state by so called Noisy Operations; localizable information $I_{l}$ is equal to the number of pure product states we can distill by CLOCC operation in the asymptotic regime. These two quantities can also be interpreted in terms of work we can draw from a heat bath by use of a given quantum state. Quantum deficit in the asymptotic regime inherits this clean operational meaning. But, on the other hand, it is much harder to evaluate. Some bounds on the regularized quantum deficit have been obtained in [8]. In our case we consider one copy version of quantum deficit and additionally we allow only for a special kind of protocols of localizing information into a subsystem. In spite of such strong restrictions we get quite interesting result.

\section{BASIC NOTION}

Informational content I of a state. Information is an abstract concept. Here we will use this term to refer to a specific function $I$

$$
I(\varrho)=N-S(\varrho)
$$

where $\varrho$ is a state of $N$ qubits, and $S(\varrho)=-\operatorname{Tr} \varrho \log \varrho$ is the von Neumann entropy. The information $I$ has oper- 
ational meaning in the asymptotic regime of many identical copies [9]. This is the unique function (up to constants) that is not increasing under the class of so called Noisy Operations (NO) [10]: operations that consist of (i) unitary transformations, (ii) partial trace, and (iii) adding ancilla in maximally mixed state. One can then show that $I$ determines the optimal rate of transitions between states under NO.

By CLOCC operations on bipartite system of $n_{A B}$ qubits we mean all operations that can be composed out of

(i) local unitary transformations,

(ii) sending a subsystem down completely decohering (dephasing) channel.

Notice that CLOCC class is a subclass of LOCC operations (Local Operations and Classical Communication) and is equivalent to local measurements, local unitary operations and classical communication. The restrictions of CLOCC (in contrast to LOCC we are forbidden to add pure ancillas and discard a local subsystem) are a consequence of the fact that we must control information flow. Every state (besides maximally mixed ones) has nonzero value of $I$, so especially pure ancillas.

Localizable information $I_{l}\left(\varrho_{A B}\right)$ of a state $\varrho_{A B}$ is the maximal amount of local information that can be obtained by CLOCC operations.

$$
I_{l}\left(\varrho_{A B}\right)=\sup _{\Lambda \in C L O C C}\left(I\left(\varrho_{A}^{\prime}\right)+I\left(\varrho_{B}^{\prime}\right)\right)
$$

where $\varrho_{A B}^{\prime}=\Lambda\left(\varrho_{A B}\right)$.

Local information $I_{L O}$ is the difference between the number of qubits $N$ of a state and the sum of entropies of its subsystems

$$
I_{L O}\left(\varrho_{A B}\right)=N-S_{A}\left(\varrho_{A B}\right)-S_{B}\left(\varrho_{A B}\right)
$$

Mutual information $I_{M}$ of a state $\varrho_{A B}$ is the difference between the sum of local entropies and the entropy of the state.

$$
I_{M}\left(\varrho_{A B}\right)=S_{A}\left(\varrho_{A B}\right)+S_{B}\left(\varrho_{A B}\right)-S\left(\varrho_{A B}\right)
$$

\section{QUANTUM AND CLASSICAL DEFICIT ZERO WAY}

In this section we introduce the quantities which are fundamental for our consideration: quantum deficit and classical deficit.

Definition 1 The quantum deficit $\Delta\left(\varrho_{A B}\right)$ of a state $\varrho_{A B}$ is given by the difference between informational content $I$ of the state and localizable information $I_{l}$

$$
\Delta\left(\varrho_{A B}\right)=I\left(\varrho_{A B}\right)-I_{l}\left(\varrho_{A B}\right)
$$

Notice that quantum deficit can be rewritten as

$$
\Delta\left(\varrho_{A B}\right)=\inf _{\Lambda \in C L O C C} S\left(\varrho_{A B}^{\prime}\right)-S\left(\varrho_{A B}\right)
$$

where $\varrho_{A B}^{\prime}=\Lambda\left(\varrho_{A B}\right)$ and $\Lambda$ is optimized over CLOCC operations.

Quantum deficit tells us about the amount of information that cannot be localized into a subsystem. It means that part of information is necessarily destroyed in the process of localizing information by use of a classical channel. So this part must be somehow quantum and come from correlations. This is the reason why we interpret quantum deficit as a measure of quantumness of correlations.

Definition 2 The classical deficit $\Delta_{c l}\left(\varrho_{A B}\right)$ of a quantum state is the difference between the information that can be localized by means of CLOCC operations (i.e. localizable information $I_{l}$ ) and local information

$$
\Delta_{c l}\left(\varrho_{A B}\right)=I_{l}\left(\varrho_{A B}\right)-I_{L O}\left(\varrho_{A B}\right)
$$

Classical deficit tells us how much more information can be obtained from a state $\varrho_{A B}$ by exploiting additional correlations in the state using a classical channel.

Notice that quantum and classical deficit add up to mutual information $I_{M}$.

$$
\Delta+\Delta_{c l}=I_{M}
$$

Thus we can express classical deficit as follows

$$
\Delta_{c l}=I_{M}-\Delta
$$

We can restrict classical communication between Alice and Bob to one-way communication (from Alice to Bob or from Bob to Alice) or to so called zero-way communication.

Consider zero-way protocol $\mathcal{P}^{\emptyset}$ of localizing information consisting of complete local measurement (or local complete dephasing) and classical communication which is allowed to be performed only after making measurement (or dephasing). Classical communication is necessary to collect data and exploit the pure classical correlations in order to localize information. Notice that the main difference between a zero-way protocol and a general CLOCC protocol of localizing information is that we are forbidden to communicate classically before all measurement or dephasing operations are finished. This guaranties that we are not able to draw any nonclassical information from correlations in a state, for example, from correlations of subsystems of some separable states which we can call classical correlations between quantum properties of the state.

If we restrict classical communication in localizable information $I_{l}$ to zero-way protocols $\mathcal{P}^{\emptyset}$ we get localizable information zero-way $I_{l}^{\emptyset}$

$$
I_{l}^{\emptyset}\left(\varrho_{A B}\right)=\sup _{\Lambda \in \mathcal{P}^{\emptyset}}\left(I\left(\varrho_{A}^{\prime}\right)+I\left(\varrho_{B}^{\prime}\right)\right)
$$

Now we can define quantum deficit zero-way $\Delta^{\emptyset}$ and classical deficit zero-way $\Delta_{c l}^{\emptyset}$. 
Definition 3 The quantum deficit zero-way $\Delta^{\emptyset}\left(\varrho_{A B}\right)$ of a state $\varrho_{A B}$ is given by the difference between informational content $I$ of the state and information $I_{l}^{\emptyset}$ localizable by zero-way protocol $\mathcal{P}^{\emptyset}$

$$
\Delta^{\emptyset}\left(\varrho_{A B}\right)=I\left(\varrho_{A B}\right)-I_{l}^{\emptyset}\left(\varrho_{A B}\right)
$$

Equivalently, we can express quantum deficit zero-way as

$$
\Delta^{\emptyset}\left(\varrho_{A B}\right)=\inf _{\Lambda \in \mathcal{P}^{\emptyset}} S\left(\varrho_{A B}^{\prime}\right)-S\left(\varrho_{A B}\right)
$$

where $\varrho_{A B}^{\prime}=\Lambda\left(\varrho_{A B}\right)$ and $\Lambda$ is optimized over $\mathcal{P}^{\emptyset}$ protocols. One can also find that quantum deficit zero-way $\Delta^{\emptyset}$ is equal to relative entropy distance from the set of classically correlated states [1].

The quantum deficit zero-way is an independent candidate for measure of quantumness of correlations. States having no quantum correlations should have $\Delta^{\emptyset}=0$. Such restricted measure can capture interesting aspects of nonlocality. Consider for example a bipartite state with eigenbasis of the form

$$
\begin{aligned}
& |0\rangle_{A}|0\rangle_{B},|0\rangle_{A}|1\rangle_{B}, \\
& \quad \frac{1}{\sqrt{2}}|1\rangle_{A}(|0\rangle+|1\rangle)_{B}, \frac{1}{\sqrt{2}}|1\rangle_{A}(|0\rangle-|1\rangle)_{B}
\end{aligned}
$$

such basis is locally indistinguishable and is also not distinguishable by zero-way communication. Therefore a mixture of the states (14) where the mixing probabilities are all different from each other would have nonvanishing $\Delta^{\emptyset}$ [1]. This is in contrast to states which are mixtures of the set of states

$$
|0\rangle_{A}|0\rangle_{B},|0\rangle_{A}|1\rangle_{B},|1\rangle_{A}|0\rangle_{B},|1\rangle_{A}|1\rangle_{B}
$$

for which all the information is extractable from the state locally, by measurement by both the parties without any communication. It suggests that the quantum behaviour of correlations could result from distinctly quantum but "local" properties of nonorthogonality. This is connected with examples of LOCC-indistinguishability of orthogonal product basis [2, 11].

In analogy to the quantum deficit zero-way $\Delta^{\emptyset}$ we can define the classical deficit zero-way $\Delta_{c l}^{\emptyset}$.

Definition 4 The classical deficit zero-way $\Delta_{c l}^{\emptyset}\left(\varrho_{A B}\right)$ of a state $\varrho_{A B}$ is the difference between information $I_{l}^{\emptyset}$ localizable by zero-way protocol $\mathcal{P}^{\emptyset}$ and local information

$$
\Delta_{c l}^{\emptyset}\left(\varrho_{A B}\right)=I_{l}^{\emptyset}\left(\varrho_{A B}\right)-I_{L O}\left(\varrho_{A B}\right)
$$

or equivalently we have that

$$
\Delta_{c l}^{\emptyset}=I_{M}-\Delta^{\emptyset}
$$

Classical deficit zero-way tells us about the amount of information that can be localized into a subsystem from correlations which are not destroyed after local measurement (or dephasing). Notice that after measurement a

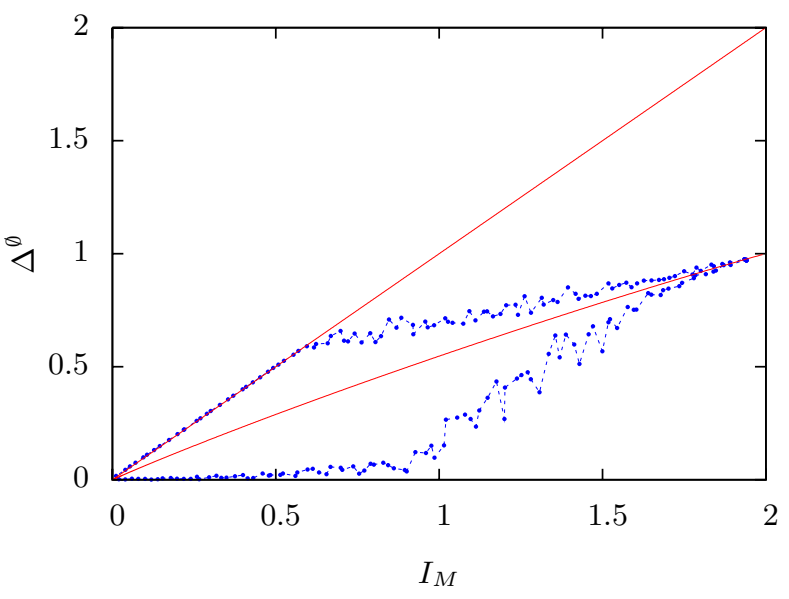

FIG. 1: Deficit zero-way is plotted versus mutual information for 100000 random two qubit states, only maximal and minimal values of $\Delta^{\emptyset}$ are plotted in short intervals of $I_{M}$. The upper line is an upper bound for $\Delta^{\emptyset}$, while the lower one is $\Delta^{\emptyset}$ of isotropic states.

state changes into classically correlated one (1) and then all information present in correlations of such a state can be localized.

There is a question how great can be quantum deficit. We know that for a pure state $\psi$ it is given by

$$
\Delta(\psi)=\frac{1}{2} I_{M}(\psi)
$$

So in particular for maximally entangled state $\psi^{+}$we have $\Delta\left(\psi^{+}\right)=\frac{1}{2} I_{M}\left(\psi^{+}\right)$. Additionally for pure states $\Delta^{\emptyset}=\Delta$, because the greatest value of localizable information is equal to the information which can be concentrated to a subsystem using $\mathcal{P}^{\emptyset}$ protocol. We can ask if there exist mixed states for which the rate of quantum deficit to $I_{M}$ is greater than for maximally entangled states, so if there are states for which amount of quantumness of correlations is greater than $\frac{1}{2} I_{M}$. We are able to answer this question for quantum deficit zero-way. What is more we can find such states for which $\Delta^{\emptyset}=I_{M}$. It implies immediately that for these states $\Delta_{c l}^{\emptyset}=0$. This means that after optimal local measurement (or dephasing) all correlations are destroyed, so by $\mathcal{P}^{\emptyset}$ protocol we cannot extract any additional information than in local scenario.

On figure 1we can see that there exist states for which $\Delta^{\emptyset}$ is equal or almost equal to $I_{M}$. So there is a task to find such states for which this equality holds.

\section{STATES FOR WHICH $\Delta^{\emptyset}=I_{M}$}

In this section we introduce a class of states $\varrho_{a}$ parameterized with $a$ and prove that these states up to some value of parameter $a$ fulfill the condition that $\Delta^{\emptyset}\left(\varrho_{a}\right)=I_{M}\left(\varrho_{a}\right)$ 


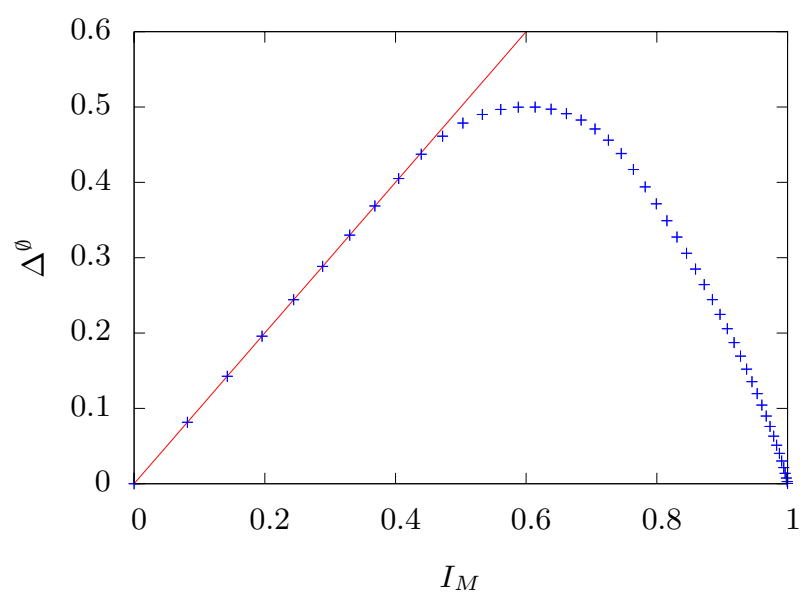

FIG. 2: Deficit zero-way is plotted versus mutual information for the class of states $\varrho_{a}$. The line is an upper bound for $\Delta^{\emptyset}$.

\section{A. Defining the class of states}

Let us define the class of states $\varrho_{a}$ for $a \in[0,1]$ on $2 \otimes 2$ Hilbert space as

$$
\varrho_{a}=\frac{1}{2}\left(\left|\phi_{a}\right\rangle\left\langle\phi_{a}|+| \psi_{a}\right\rangle\left\langle\psi_{a}\right|\right)
$$

where

$$
\begin{aligned}
& \left|\phi_{a}\right\rangle=\sqrt{a}|00\rangle+\sqrt{1-a}|11\rangle \\
& \left|\psi_{a}\right\rangle=\sqrt{a}|01\rangle+\sqrt{1-a}|10\rangle
\end{aligned}
$$

In our problem $\varrho_{a}$ and $\varrho_{1-a}$ are equivalent (they only differ by a local operation) thus we will only consider $a \in\left[0, \frac{1}{2}\right]$.

The mutual information for states $\varrho_{a}$ is given by

$$
I_{M}\left(\varrho_{a}\right)=H(a)
$$

thus the value of $I_{M}$ may be freely chosen in the interval $[0,1]$ by proper selection of parameter $a$.

The deficit zero-way for states $\varrho_{a}$ is given by

$$
\Delta^{\emptyset}\left(\varrho_{a}\right)=\inf _{\Lambda \in \mathcal{P}^{\emptyset}} S\left(\varrho_{a}^{\prime}\right)-1
$$

Numerical deficit zero-way versus mutual information for the states of our class $\varrho_{a}$ suggests (figure 2) that for some interval $\left[0, a_{0}\right]$ of parameter $a$ we have equality between quantities $\Delta^{\emptyset}$ and $I_{M}$.

\section{B. Sketch of the proof}

To optimize the value of $\Delta^{\emptyset}$ given by (22) we start from the formula for $S\left(\varrho^{\prime}\right)$ reachable under $\mathcal{P}^{\emptyset}$ protocols. Then we reduce the optimization of $S\left(\varrho^{\prime}\right)$ to the optimization of single real parameter function $S_{\alpha}\left(\gamma_{A}\right)$, where $\alpha$ is determined by $a$. Later by analyzing the first and second order derivatives of $S_{\alpha}\left(\gamma_{A}\right)$ we argue that for $\alpha \in\left[\alpha_{0}, \frac{\pi}{2}\right]$ corresponding to $a \in\left[0, a_{0}\right]$ there is the global minimum of $S_{\alpha}\left(\gamma_{A}\right)$ in $\gamma_{A}=0$ which implies $\Delta^{\emptyset}=I_{M}$.

On the other hand for $\alpha \in\left[0, \alpha_{0}\right)$ corresponding to $a \in\left(a_{0}, \frac{1}{2}\right]$ there is a maximum of $S_{\alpha}\left(\gamma_{A}\right)$ in $\gamma_{A}=0$ which implies $\Delta^{\emptyset}<I_{M}$. Finally we compute the value of $a_{0}$.

\section{Helpful functions}

First we introduce two functions, which will be useful in the further consideration. Let us define

$$
\begin{aligned}
& H_{s}(x)=H\left(\frac{1+\sin (x)}{2}\right) \\
& H_{c}(x)=H\left(\frac{1+\cos (x)}{2}\right)
\end{aligned}
$$

The above functions have the following properties

1. $H_{s}$ and $H_{c}$ are even so their first derivatives are odd and the second are even.

2. $H_{s}$ and $H_{c}$ are periodic with period $\pi$ and so are derivatives.

3. $H_{s}\left(\frac{\pi}{2} \pm x\right)=H_{c}(x)$ and similar for derivatives.

\section{Simplifying the form of $S\left(\varrho_{a}^{\prime}\right)$}

For any two qubit state $\varrho_{a}^{\prime}$ reachable under $\mathcal{P}^{\emptyset}$ protocol its von Neumann entropy can be expressed by

$$
S\left(\varrho_{a}^{\prime}\right)=H\left(\operatorname{diagonal}\left(U_{A} \otimes U_{B} \varrho_{a} U_{A}^{\dagger} \otimes U_{B}^{\dagger}\right)\right)
$$

where $U_{A}$ and $U_{B}$ are single qubit unitaries which can be parameterized as follows [12]:

$$
U=e^{i \alpha}\left[\begin{array}{cc}
e^{i\left(-\frac{\beta}{2}-\frac{\delta}{2}\right)} \cos \left(\frac{\gamma}{2}\right) & -e^{i\left(-\frac{\beta}{2}+\frac{\delta}{2}\right)} \sin \left(\frac{\gamma}{2}\right) \\
e^{i\left(\frac{\beta}{2}-\frac{\delta}{2}\right)} \sin \left(\frac{\gamma}{2}\right) & e^{i\left(\frac{\beta}{2}+\frac{\delta}{2}\right)} \cos \left(\frac{\gamma}{2}\right)
\end{array}\right]
$$

Using this parameterization for $U_{A}$ and $U_{B}$ we can simplify with help of Maxima 14] the form of $S\left(\varrho_{a}^{\prime}\right)$ to

$$
S\left(\varrho_{a}^{\prime}\right)=H\left(\frac{1+s-c}{4}, \frac{1-s-c}{4}, \frac{1-s+c}{4}, \frac{1+s+c}{4}\right)
$$

where

$$
\begin{aligned}
& s=2 \sqrt{a(1-a)} \sin \gamma_{A} \sin \gamma_{B} \cos \delta_{A} \cos \delta_{B} \\
& c=(1-2 a) \cos \gamma_{A}
\end{aligned}
$$

(We subscript the parameters with the name of the subsystem). Note that this form of $S\left(\varrho_{a}^{\prime}\right)$ exhibits asymmetry with respect to parameters of $U_{A}$ and $U_{B}$. Our class of states $\varrho_{a}$ is indeed asymmetric (i.e. $\varrho_{a} \neq V \varrho_{a} V$ where $V$ is the swap operator) unless $a=\frac{1}{2}$, for example, $S_{A}\left(\varrho_{a}\right) \neq S_{B}\left(\varrho_{a}\right)$. The asymmetry with respect to parameters of $U_{A}$ and $U_{B}$ will appear even stronger after the next simplification.

Let us here recall the fact: 
Fact 1 Any change toward equalization of probabilities $p_{1}, p_{2}, \ldots, p_{n}$ increases $H$. Thus if $p_{1}<p_{2}$ and we increase $p_{1}$, decreasing $p_{2}$ an equal amount so that $p_{1}$ and $p_{2}$ are more nearly equal, then $H$ increases. (...) [1]]

We group probabilities from distribution (27) in two inequalities

$$
\frac{(1 \pm c)-|s|}{4} \leq \frac{(1 \pm c)+|s|}{4}
$$

Now we can see that decreasing $|s|$ for any fixed value of $c$ will change both inequalities towards equalization and hence from fact 1 will increase entropy (27). As we are minimizing $S\left(\varrho_{a}^{\prime}\right)$ in (22) thus we should maximize $|s|$ in respect to the parameters with no influence on $c$. We can do this by setting

$$
\sin \gamma_{B} \cos \delta_{A} \cos \delta_{B}=1
$$

in (28). After this substitution we observe that $s+c$ and $s-c$ are harmonic oscillations, i.e.

$$
s \pm c=\sin \left(\gamma_{A} \pm \alpha\right)
$$

where $\alpha$ is determined by

$$
\begin{aligned}
\sin \alpha & =1-2 a \\
\cos \alpha & =2 \sqrt{a(1-a)}
\end{aligned}
$$

The harmonic oscillations of (32) allow us to simplify $S\left(\varrho_{a}^{\prime}\right)$ given by (27) to

$$
S_{\alpha}\left(\gamma_{A}\right)=1+\frac{1}{2} H_{s}\left(\gamma_{A}+\alpha\right)+\frac{1}{2} H_{s}\left(\gamma_{A}-\alpha\right)
$$

with the property that

$$
\inf _{\Lambda \in \mathcal{P}^{\emptyset}} S\left(\varrho_{a}^{\prime}\right)=\inf _{\gamma_{A} \in\left[-\frac{\pi}{2}, \frac{\pi}{2}\right]} S_{\alpha}\left(\gamma_{A}\right)
$$

\section{E. Proving $\Delta^{\emptyset}=I_{M}$ for $a \in\left[0, a_{0}\right]$}

First we observe that

$$
S_{\alpha}(0)=1+H(a)
$$

thus if $\gamma_{A}=0$ is the global minimum of $S_{\alpha}\left(\gamma_{A}\right)$ for some $\alpha$ we get the desired equality

$$
\Delta^{\emptyset}\left(\varrho_{a}\right)=(1+H(a))-1=I_{M}\left(\varrho_{a}\right)
$$

On the other hand if $\gamma_{A}=0$ is a maximum of $S_{\alpha}\left(\gamma_{A}\right)$ for some $\alpha$ than the value of $S_{\alpha}\left(\gamma_{A}\right)$ in the global minimum is less than $S_{\alpha}(0)$ and thus $\Delta^{\emptyset}<I_{M}$.

In search for extrema of $S_{\alpha}\left(\gamma_{A}\right)$ we consider its derivative

$$
\frac{\mathrm{d} S_{\alpha}\left(\gamma_{A}\right)}{\mathrm{d} \gamma_{A}}=\frac{1}{2}\left(\frac{\mathrm{d} H_{s}\left(\gamma_{A}+\alpha\right)}{\mathrm{d} \gamma_{A}}+\frac{\mathrm{d} H_{s}\left(\gamma_{A}-\alpha\right)}{\mathrm{d} \gamma_{A}}\right)
$$

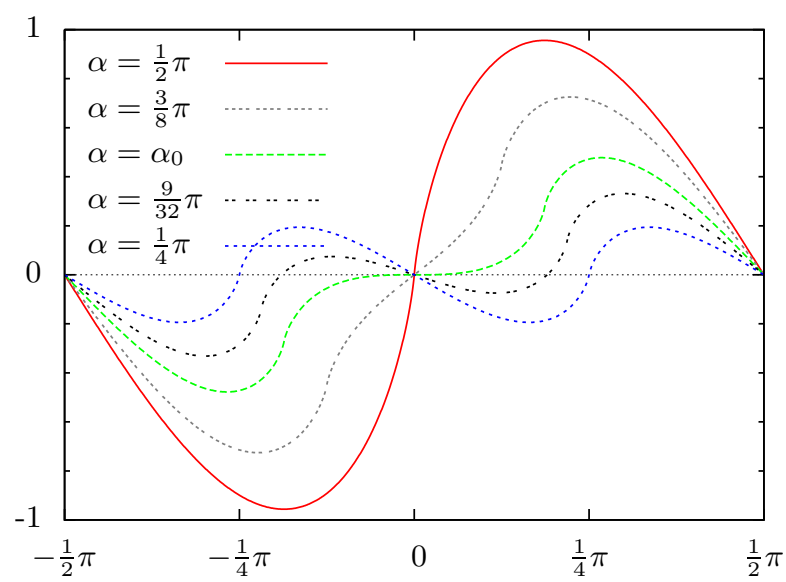

FIG. 3: First order derivative of $S_{\alpha}\left(\gamma_{A}\right)$ for sample values of $\alpha$ illustrating the appearance of two more zeros.

Notice that $S_{\alpha}\left(\gamma_{A}\right)$, same as $H_{s}$, is periodic with period $\pi$. Thus it is enough to consider the range of a single period $\gamma_{A} \in\left[-\frac{\pi}{2}, \frac{\pi}{2}\right]$. We identify both ends of the period when it comes to listing zeros in a period. In the interval $\left[-\frac{\pi}{2}, \frac{\pi}{2}\right]$ the derivative of $S_{\alpha}\left(\gamma_{A}\right)$ has for all $\alpha$ zeros in $\gamma_{A}=0$ and $\gamma_{A}=\frac{\pi}{2}$ (as derivatives of $H_{s}$ and $H_{c}$ are odd; figure 3).

We also consider the second order derivative of $S_{\alpha}\left(\gamma_{A}\right)$ which is the average of two impulses relatively shifted by $2 \alpha$. By the impulse we mean a function of the form

$$
\frac{\mathrm{d}^{2} H_{s}(x)}{\mathrm{d} x^{2}}=\frac{1}{2} \sin (x) \cdot(\log \circ f \circ \sin )(x)-\frac{1}{\ln 2}
$$

where

$$
f(x)=\frac{1+x}{1-x}
$$

Since $f$ is strictly increasing in the interval $[-1,1)$, thus $f \circ \sin$ is strictly increasing in $\left[0, \frac{\pi}{2}\right)$ and strictly decreasing in $\left(-\frac{\pi}{2}, 0\right]$ and the same holds for $\log \circ f \circ \sin$ and the impulse. The impulse has a peak in $\frac{\pi}{2}$ by which we mean it tends to infinity in this point. The impulse also has one negative and one positive interval per period. It can be observed on figure 4 as the curve labeled $\alpha=0$.

Now we analyze the extrema of $S_{\alpha}\left(\gamma_{A}\right)$ for $\alpha \in\left[0, \frac{\pi}{2}\right]$, which corresponds to $a \in\left[0, \frac{1}{2}\right]$.

For $\alpha=\frac{\pi}{2}$ corresponding to $a=0$ both impulses of the second order derivative of $S_{\alpha}\left(\gamma_{A}\right)$ are equal and have peaks in $\gamma_{A}=0$ (figure 44) and thus $\gamma_{A}=0$ is a minimum of $S_{\alpha}\left(\gamma_{A}\right)$. If we move with $\alpha$ from $\frac{\pi}{2}$ down to 0 than peaks of both impulses are shifting away from $\gamma_{A}=0$ and thus the value of the second order derivative of $S_{\alpha}\left(\gamma_{A}\right)$ in $\gamma_{A}=0$ is strictly monotonically decreasing to $(-\ln 2)^{-1}$ for $\alpha=0$. The point in which it reaches 0 on this way will be called $\alpha_{0}$.

Thus for $\alpha \in\left[0, \alpha_{0}\right)$ corresponding to $a \in\left(a_{0}, \frac{1}{2}\right]$ the second order derivative is negative in $\gamma_{A}=0$ and so $S_{\alpha}\left(\gamma_{A}\right)$ has maximum in $\gamma_{A}=0$ which implies $\Delta^{\emptyset}<I_{M}$. 


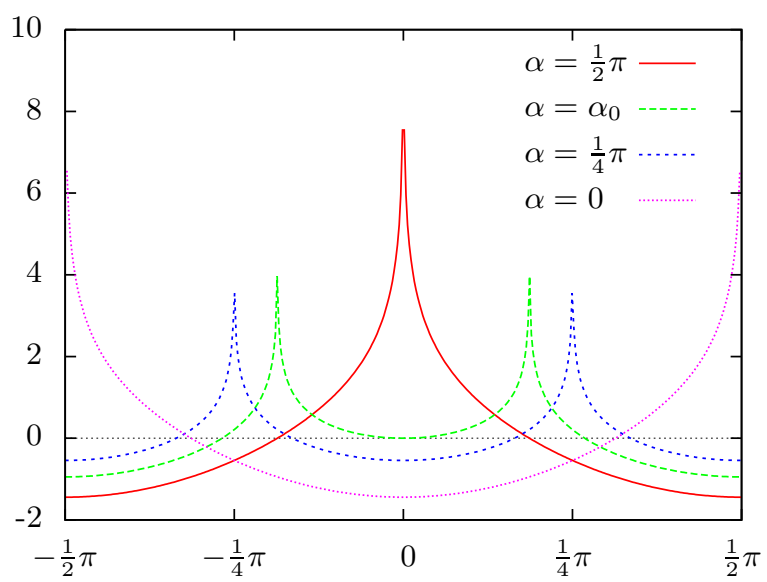

FIG. 4: Second order derivative of $S_{\alpha}\left(\gamma_{A}\right)$ for the most characteristic values of the range $\alpha \in\left[0, \frac{\pi}{2}\right]$.

For $\alpha \in\left[\alpha_{0}, \frac{\pi}{2}\right]$ corresponding to $a \in\left[0, a_{0}\right]$ the second order derivative of $S_{\alpha}\left(\gamma_{A}\right)$ has one negative and one positive interval per period which implies that the first order derivative has only one strictly increasing and one strictly decreasing interval per period and thus at most two zeros (one per interval). So it has exactly two zeros: $\gamma_{A}=0$ and $\gamma_{A}=\frac{\pi}{2}$, which must appear for all $\alpha$. For $\alpha \in\left[\alpha_{0}, \frac{\pi}{2}\right]$ the first order derivative of $S_{\alpha}\left(\gamma_{A}\right)$ is negative in $\gamma_{A}=\frac{\pi}{2}$ thus $\gamma_{A}=\frac{\pi}{2}$ is the global maximum of $S_{\alpha}\left(\gamma_{A}\right)$ which implies $\gamma_{A}=0$ is the global minimum and so $\Delta^{\emptyset}=I_{M}$ for $a \in\left[0, a_{0}\right]$.

The last step is to find $a_{0}$.

\section{F. Finding $a_{0}$}

To find $a_{0}$ we numerically solve the equation

$$
\left.\frac{\mathrm{d}^{2} S_{\alpha}\left(\gamma_{A}\right)}{\mathrm{d} \gamma_{A}^{2}}\right|_{\gamma_{A}=0}=(1-2 a) \operatorname{arctanh}(1-2 a)-1=0
$$

and the smaller of two roots gives $a_{0}$

$$
a_{0} \approx 0.08322
$$

\section{REACHING LOWER BOUND ON $\Delta^{\emptyset}$ FOR A GIVEN $I_{M}$}

On $2 \otimes 2$ Hilbert space $\Delta_{c l}^{\emptyset} \leq 1$ thus on this space the lower bound on $\Delta^{\emptyset}$ is a function of $I_{M}$

$$
\Delta^{\emptyset} \geq \max \left(0, I_{M}-1\right)
$$

We show that for any $I_{M}$ this lower bound is achievable.

For $I_{M} \in[0,1]$ the lower bound is achieved by the simple class of separable states

$$
\sigma_{p}=p|00\rangle\langle 00|+(1-p)| 11\rangle\langle 11|
$$

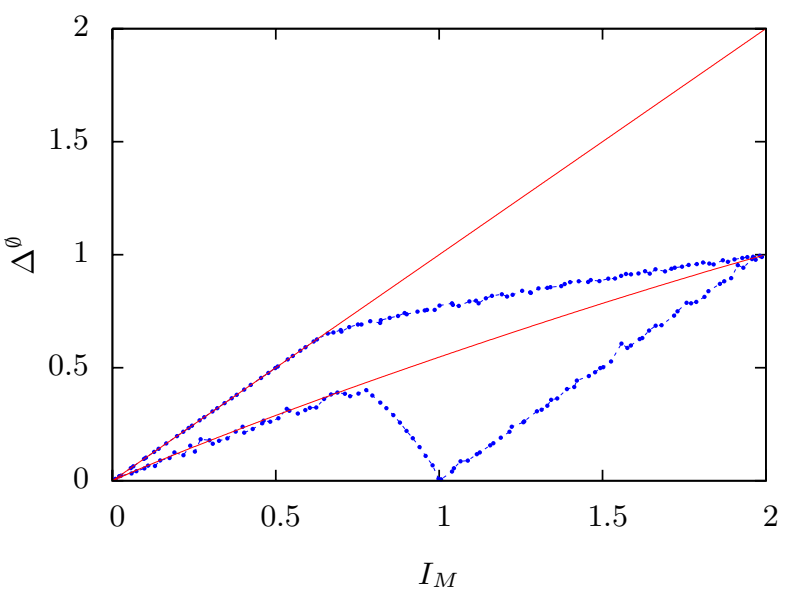

FIG. 5: Deficit zero-way is plotted versus mutual information for 10000 random two qubit states from $\varrho_{a, b, p}$ class, only maximal and minimal values of $\Delta^{\emptyset}$ are plotted in short intervals of $I_{M}$. The upper line is an upper bound for $\Delta^{\emptyset}$, while the lower one is $\Delta^{\emptyset}$ of isotropic states.

While for $I_{M} \in[1,2]$ the lower bound is achieved by the class of states

$$
\varrho_{p}=p\left|\phi^{+}\right\rangle\left\langle\phi^{+}|+(1-p)| \psi^{+}\right\rangle\left\langle\psi^{+}\right|
$$

where

$$
\begin{aligned}
\left|\phi^{+}\right\rangle & =\frac{1}{\sqrt{2}}(|00\rangle+|11\rangle) \\
\left|\psi^{+}\right\rangle & =\frac{1}{\sqrt{2}}(|01\rangle+|10\rangle)
\end{aligned}
$$

For $\sigma_{p}$ the infimum of $\Delta^{\emptyset}$ as given by (13) is achieved by setting $U_{A}=U_{B}=I$ in (25) while for $\varrho_{p}$ by $U_{A}=$ $U_{B}=H$, where $H$ is the Hadamard gate.

\section{ANOTHER INTERESTING CLASS}

Let us introduce another class of states on $2 \otimes 2$ Hilbert space

$$
\varrho_{a, b, p}=p\left|\phi_{a}\right\rangle\left\langle\phi_{a}|+(1-p)| \psi_{b}\right\rangle\left\langle\psi_{b}\right|
$$

where $\left|\phi_{a}\right\rangle$ and $\left|\psi_{b}\right\rangle$ are given by (19) and (20) respectively. This class is a generalization of two of previously considered classes, i.e.

$$
\begin{aligned}
& \varrho_{a}=\varrho_{a, a, \frac{1}{2}} \\
& \varrho_{p}=\varrho_{\frac{1}{2}, \frac{1}{2}, p}
\end{aligned}
$$

Random elements of the $\varrho_{a, b, p}$ class (figure 5) cover wider range of $\Delta^{\emptyset}$ than general two qubit states (figure $\mathbb{1}$ ), except some evident region, although there are 100000 states on figure 1 and only 10000 on figure 5. 
What is interesting, for $\varrho_{a, b, p}$ states the condition $\Delta^{\emptyset}=I_{M}$ require $\varrho_{a, b, p}$ to be a separable state. It comes from the fact that if we want to fulfill this condition then after measurement made in eigenbasis of subsystems of a state we have to obtain a product state. Otherwise we would be able to get more than $2-S_{A}-S_{B}$ localizable information zero-way, because $S\left(\varrho^{\prime}\right)<S_{A}+S_{B}$ for nonproduct states. The above condition for states $\varrho_{a, b, p}$ can be expressed by the parameters $a, b, p$ as follows

$$
p^{2} a(a-1)-(1-p)^{2} b(1-b)=0
$$

which is equivalent to

$$
p \sqrt{a(a-1)}=(1-p) \sqrt{b(1-b)}
$$

And equality (53) implies via partial transposition criterion that $\varrho_{a, b, p}$ is a separable state.

\section{ENTANGLED STATES FOR WHICH $\Delta^{\emptyset}=I_{M}$ MAY HOLD}

Consider the following class of states (mixtures of two nonorthogonal states) on $2 \otimes 2$ Hilbert space

$$
\sigma_{a}=\frac{1}{2}\left(\frac{|u\rangle\langle u|}{\|u\|}+\frac{\left|v_{a}\right\rangle\left\langle v_{a}\right|}{\left\|v_{a}\right\|}\right)
$$

where

$$
\begin{aligned}
|u\rangle & =|00\rangle+2|11\rangle \\
\left|v_{a}\right\rangle & =|00\rangle+a|01\rangle-2|10\rangle-2|11\rangle
\end{aligned}
$$

For the state $\sigma_{a}$ to have product diagonal in eigenbasis of its subsystems (which is the necessary condition of $I_{M}=\Delta^{\emptyset}$ as shown in previous section) the parameter $a$ must satisfy the equation

$$
\left(4 a^{4}+40 a^{3}+87 a^{2}+160 a-341\right) R(a)=0,
$$

where $R(a)$ is some rational expression. The polynomial of degree 4 has two real solutions, one around -8.1 and one around 1.1. Both give entangled states but the negative one has $I_{M}-\Delta^{\emptyset}>0.09$. The positive one is

$$
\begin{aligned}
a_{0} & =\frac{\sqrt{7200 \sqrt{z}-\sqrt{w}\left(w-504 z^{\frac{1}{3}}\right)}}{8 w^{\frac{1}{4}} z^{\frac{1}{6}}}-\frac{\sqrt{w}}{8 z^{\frac{1}{6}}}-\frac{5}{2} \\
& \approx 1.10122
\end{aligned}
$$

where

$$
\begin{aligned}
& w=16 z^{\frac{2}{3}}+168 z^{\frac{1}{3}}-3111 \\
& z=\frac{75 \sqrt{527523}}{16}-\frac{131787}{64}
\end{aligned}
$$

The state $\sigma_{a_{0}}$ is entangled and satisfies the required condition of equality and numerical optimizations and parameterized plots suggest it may fulfill the $I_{M}=\Delta^{\emptyset}$ equality.

If we generalize $\sigma_{a}$ to $\sigma_{a, t}$ where instead of $\left|v_{a}\right\rangle$ we take

$$
\left|v_{a, t}\right\rangle=|00\rangle+a|01\rangle-t|10\rangle-t|11\rangle
$$

it seems that for all $t$ starting with some $t_{0}\left(1<t_{0}<2\right)$ we can find $a_{t}$ with product diagonal in eigenbasis of subsystems and $I_{M}=\Delta^{\emptyset}$ (or almost equal). So $\sigma_{a_{t}, t}$ may be (for some range of $t$ ) a class of entangled states satisfying the equality of $I_{M}=\Delta^{\emptyset}$.

\section{SUMMARY}

In our paper we showed that there are states for which quantum correlations are completely quantum. We made this by presenting a family of states for which mutual information (the measure of total correlations) is equal to quantum deficit zero-way (a measure of quantumness of correlations). Surprisingly, the states which we have found are separable. We also presented numerical results which suggest that such situation is possible for entangled states. What is intriguing we know that the optimal protocol $\mathcal{P}^{\emptyset}$ which achieves the value of mutual information is made in local eigenbasis of subsystems and gives as a result a product state of the form $\varrho_{A} \otimes \varrho_{B}$, where $\varrho_{A}$ and $\varrho_{B}$ are states of subsystems. This is equivalent to localizing only local information. Any other local measurement gives us nonproduct classical states, from which we are able to localize whole its global information but still less then by only local action. So the price of producing classical correlations from which we can get additional information is too high. The produced entropy is greater than gained information.

\section{ACKNOWLEDGEMENTS}

We would like to thank Michał Horodecki and Ryszard Horodecki for helpful discussions. This work is supported by EU grant SCALA FP6-2004-IST no.015714 and by Polish Ministry of Science and Education under the (solicited) grant No. PBZ-MIN-008/P03/2003. 
[1] M. Horodecki, P. Horodecki, R. Horodecki, J. Oppenheim, A. Sen(De), U. Sen, and B. Synak-Radtke, Phys. Rev. A 71, 062307 (2005), quant-ph/0410090.

[2] C. H. Bennett, D. P. DiVincenzo, C. A. Fuchs, T. Mor, E. Rains, P. W. Shor, J. Smolin, and W. K. Wootters, Phys. Rev. A 59, 1070 (1999), quant-ph/9804053.

[3] H. Ollivier and W. H. Zurek, Phys. Rev. Lett 99, 017901 (2001), quant-ph/0105072.

[4] W. H. Zurek, Phys. Rev. A 67, 012320 (2003), quant$\mathrm{ph} / 0202123$.

[5] J. Oppenheim, M. Horodecki, P. Horodecki, and R. Horodecki, Phys. Rev. Lett 89, 180402 (2002), quant$\mathrm{ph} / 0112074$.

[6] B. Groisman, D. Kenigsberg, and T. Mor (2007), quant$\mathrm{ph} / 0703103$.

[7] A. SaiToh, R. Rahimi, and M. Nakahara, quant$\mathrm{ph} / 0703133$.

[8] B. Synak-Radtke, K. Horodecki, and M. Horodecki, J.
Math. Phys. 46, 082107 (2005), quant-ph/0405149.

[9] M. Horodecki, K.Horodecki, P.Horodecki, R. Horodecki, J. Oppenheim, A. Sen(De), and U. Sen, Phys. Rev. Lett. 90, 100402 (2003), quant-ph/0207168.

[10] M. Horodecki, P. Horodecki, and J. Oppenheim, Phys. Rev. A (2003).

[11] C. H. Bennett, D. P. DiVincenzo, T. Mor, P. W. Shor, J. A. Smolin, and B. M. Terhal, Phys. Rev. Lett. 82, 5385 (1999), quant-ph/9808030.

[12] M. A. Nielsen and I. L. Chuang, Quantum Computation and Quantum Information (Cambridge University Press, Cambridge, 2000).

[13] C. Shannon, Bell System Technical Journal 27, $379 \quad$ (1948), $\quad$ http://cm.belllabs.com/cm/ms/what/shannonday/paper.html.

[14] Maxima (http://maxima.sourceforge.net/) is a free software computer algebra system released under GNU GPL. 